\title{
Decision processes during sentence comprehension: Effects of surface structure reconsidered ${ }^{1}$
}

Previous experiments have yielded conflicting results concerning the utility of relative pronouns as cues to the deep structure underlying self-embedded (SE) sentences. The present study investigated the usefulness of this cue. At the same time, it compared the relative sen sitivity of two measures of sentence-comprehension difficulty: paraphrasing and phoneme monitoring. Forty Ss heard SE sentences, one-half of which had the relative pronouns deleted. The presence or absence of the relative pronouns in a particular sentence was counterbalanced across groups. The Ss also heard right-branching sentences. It was found that presence of the relative pronouns led to significantly faster phoneme monitoring times and to marginally better paraphrasing (i.e., to better comprehension) in the SE sentences. It was concluded that relative pronouns are effective cues and, further, that the phoneme monitoring technique is a better index of comprehension difficulty than the paraphrasing technique. Reasons for the previous experimental discrepancies were discussed.

Recent work on sentence comprehension has generally supported the hypothesis that "the complexity of a sentence is [in part] a function ... of the degree to which the arrangement of elements in the surface structure provides clues to the relations of elements in the deep structure [Fodor \& Garrett, 1967]." Some evidence in favor of the surface structure cue ${ }^{3}$ hypothesis was provided by Fodor and Garrett, who presented $S$ s with self-embedded sentences like (1) and (2) and asked them to paraphrase each sertence.

(1) The car that the man whom the dog bit drove crashed.

(2) The car the man the dog bit drove crashed.

Both Sentences 1 and 2 contain embedded relative clauses, but in (2) the relative clauses have been reduced by deleting the relative pronouns: On the basis of the surface-structure cue hypothesis, Fodor and Garrett predicted that sentences like (1) would be easier to comprehend than sentences like (2) because the Type 1 sentences contain relative pronouns which are signs that the relative clause embedding transformations have been applied and thus are cues to the underlying grammatical relations. In their experiments, Fodor and Garrett found that Type 1 sentences were paraphrased more quickly and more accurately than Type 2 sentences. We will refer to Type 1 sentences, those that contain the relative pronouns, as unreduced sentences and to Type 2 sentences as reduced sentences.

An experiment by Foss and Lynch (1969, Experiment 2) yielded data that did not corroborate Fodor and Garrett's findings. Foss and Lynch measured sentence comprehension difficulty with a "phoneme monitoring" technique. Briefly, this technique requires $S s$ to listen in each sentence they hear for a word beginning with a particular letter or phoneme (always a /b/ in the study cited) and to press a button when they hear one. Response latency is taken as a measure of the amount of processing $S$ is engaged in at the point where the target phoneme occurs (see also Foss, 1969).

In an earlier experiment, Foss and Lynch (1969, Experiment 1) showed that latency in the monitoring task was greater for self-embedded (SE) sentences than for right-branching (RB) sentences like (3) when the target phoneme occurred late in the sentence (i.e., when it was the initial phoneme of the last verb). As predicted, there was no difference when the target phoneme occurred early (i.e., in the first noun), for up to this point the $S E$ and $R B$ structures do not differ.

(3) The dog bit the man who drove the car that crashed.

In Experiment 2, Foss and Lynch extended their design to include both reduced and unreduced SE sentences. The experiment thus included $\mathrm{RB}$, unreduced $\mathrm{SE}$, and reduced $\mathrm{SE}$ sentences in which target phonemes occurred either early, late, or not at all. Ten sentences were used in each of these nine conditions in a within-Ss design. The most salient finding was that there was no difference between reduced and unreduced SE sentences for the late target phonemes. On the assumption that both the Fodor-Garrett paraphrasing task and the Foss-Lynch monitoring task reflect comprehension difficulty, this result is inconsistent both with Fodor and Garrett's data and with their surface structure cue hypothesis.

It should be noted that the issues raised by these two studies are broader than the question of whether or not reduction of relative clauses makes $S E$ sentences more difficult to comprehend. An additional, and important, issue is whether the two tasks (paraphrasing and phoneme monitoring) both reflect comprehension difficulty. It would be desirable if they did, of course, for then investigators would have two independent measures of this variable. Also, Hakes and Cairns (1970) have pointed out that the paraphrasing task is insensitive to comprehension difficulty except when difficulty is so great as to render the sentences (nearly) incomprehensible. Consequently, it is also insensitive to differences in comprehension difficulty. Other results (e.g., Foss, 1969) suggest that the phoneme monitoring task is a sensitive measure of such differences. However, no firm conclusions can be drawn concerning the relative sensitivity of the tasks as long as it is unclear that both $r \in f l e c t$ comprehension difficulty; in particular, as long as the discrepancy in results on the effects of relative clause reduction remains unexplained.

Some data relevant to this problem are available. Hakes and Cairns (1970) have reported an experiment using both the paraphrasing and monitoring tasks with SE sentences such as (1) and (2). The sentences used were taken from the Fodor and Garrett experiments and were presented, either reduced or unreduced, together with a set of filler sentences. For the SE sentences, the word containing the target phoneme was the second varb, unlike the Foss and Lynch experiment where it was the last (third) verb. In addition, the $S s$ were required to paraphrase each sentence. In contrast to the Fodor and Garrett experiment, in which Ss heard each sentence five times, Ss heard and paraphrased each sentence only once. The results indicated highly significant effects of the relative clause reduction variable for paraphrasing accuracy and for the monitoring task. Paraphrasing latency did not, however, yield the expected difference. The Hakes and Cairns results indicate that the monitoring task is capable of yielding the same effects as the paraphrasing task and provide further support for the surface structure cue hypothesis. The problem remains, however, of why Foss and Lynch failed to obtain the predicted result.

Hakes and Cairns surveyed a number of 
differences among the experiments and suggested a possible reason for the Foss and Lynch results. Foss and Lynch did not counterbalance their materials, with the result that the $\mathrm{SE}$ sentences which appeared in reduced form were the same for all $S s$ and were different from those which appeared in unreduced form. Although the reduced and unreduced sentences were randomly assigned from a single set, it seems possible that the subsets differed in some way. An examination of the sentences suggested that the verbs in the unreduced sentences were more complex (i.e., could occur in a greater variety of syntactic structures) than those in the reduced sentences. Fodor, Garrett, and Bever (1968) have shown that increased verb complexity is associated with increased comprehension difficulty. It thus seemed possible that a difference in verb complexity in Foss and Lynch's sentences countered the difference due to relative clause reduction, yielding no net difference.

The experiment reported here was designed to test the hypothesis that the failure of Foss and Lynch to obtain the relative clause reduction effect was due to the above-mentioned materials effect. In addition, it was designed to provide further information on the relation between the paraphrasing and phoneme monitoring tasks as indices of comprehension difficulty. Specifically, the experiment used the sentences from the Foss and Lynch experiment, introducing counterbalancing of the materials in the reduced and unreduced $S E$ conditions. Phoneme monitoring and paraphrasing data were both collected.

\section{Design and Materials}

\section{METHOD}

Forty Ss, drawn from introductory psychology classes, each heard the 90 sentences from the Foss and Lynch experiment. Thirty of these sentences were $\mathrm{RB}, 30$ were $\mathrm{SE}$ reduced $\left(S E_{R}\right)$, and 30 were $\mathrm{SE}$ unreduced $\left(\mathrm{SE}_{U}\right)$. Within each sentence type, the target phoneme occurred early (E), late (L), or not at all (N) an equal number of times (i.e., 10). When it occurred early, the target began the sentence's first noun; when late, it began the last verb.

The Ss were divided into two groups. For Group 1 the assignment of SE sentences to the $R$ and $U$ conditions was identical to that for the Foss and Lynch experiment, while for Group 2 the assignment was reversed. Group 1 represents a replication of the Foss and Lynch experiment, while Group 2 counterbalances the materials with respect to relative clause reduction. Two Es each

Table 1

Mean Speed Scores for the Phoneme Monitoring Task

\begin{tabular}{|c|c|c|c|c|c|c|}
\hline \multirow{2}{*}{$\begin{array}{l}\text { Target } \\
\text { Position }\end{array}$} & \multicolumn{3}{|c|}{ Group 1} & \multicolumn{3}{|c|}{ Group 2} \\
\hline & RB & SEU & $\mathrm{SE}_{\mathrm{R}}$ & $\mathbf{R B}$ & SEU & $S_{R}$ \\
\hline Early & 1.59 & 1.54 & 1.61 & 1.58 & 1.72 & 1.76 \\
\hline Late & 1.44 & 1.17 & 1.09 & 1.55 & 1.24 & 1.11 \\
\hline
\end{tabular}

tested half the Ss in each group. Thus there were two between-S variables: groups (i.e., materials) and Es. There were also two within-S variables: sentence type and target phoneme location. The design cannot be characterized as a simple factorial since the RB materials were the same for Groups 1 and 2 . Hence, the analysis procedures had to be adjusted accordingly.

If Foss and Lynch's failure to find a relative clause reduction effect was due to the materials used in their $S_{U}-\mathrm{L}$ and $S E_{R}-L$ conditions, then we would expect there to be no difference between these conditions for Group 1. There whould, however, be a substantial difference for Group 2, since the relative clause reduction effect and any materials effect should combine to yield a difference between these conditions in the same direction. Thus we predict a significant interaction between groups and reduction for the late condition.

The instructions for the phoneme monitoring and paraphrasing tasks (taken from the Foss-Lynch and Fodor-Garrett studies, respectively) and the sentences were tape recorded. Separate tapes, incorporating the counterbalanced SE sentences, were recorded for Groups 1 and 2. The order of the sentences was identical to that of the Foss-Lynch experiment. There were 10 blocks of nine sentences, one sentence of each type occurring randomly in each block. A pulse (inaudible to $S)$ was recorded on the tape, coincident with the beginning of each word containing a target phoneme. The pulses were used to start a timer used for measuring monitoring latency.

Subjects were required to perform two tasks. The first, phoneme monitoring, required them to listen in each sentence for a word beginning with $a / b /$ and, if they heard one, to press a button as quickly as possible. The second task asked them to comprehend the sentence and, as soon as it was completed, to say in their own words what it meant.

\section{Procedure}

The $S$ was seated in front of a microphone. The instructions and sentences were presented binaurally through headphones. Four practice sentences were presented at the end of the instructions, two being RB and two, SE. Following the practice sentences, questions were answered. Then $\mathrm{S}$ was presented the 90 sentences, with a 5-min rest pause occurring following Sentence 54 . Immediately after each sentence the tape recorder was stopped and $\mathrm{S}$ gave his paraphrase. He was given as much time as necessary, and the next sentence was presented approximately $3 \mathrm{sec}$ after the paraphrase was completed. The experimental session was recorded for later analysis of the paraphrases.

\section{RESULTS AND DISCUSSION}

\section{Phoneme Monitoring Task}

The response latencies were converted to speed scores by reciprocal transformation, and all analyses were performed on these scores. For each S, mean speed scores were computed for early and for late target phonemes for the RB. $S E_{U}$, and $S E_{R}$ conditions. The means of these mean speed scores for Groups 1 and 2 are presented in Table 1.

Because the relative clause reduction effect was of primary interest, the data for the reduced and unreduced $S E$ sentences were analyzed separately from those for the RB sentences. For the SE sentences, the mean speed scores were subjected to a 2 by 2 by 2 by 2 , Groups by Es by Target Position $(E, L)$ by Reduction $\left(\mathrm{SE}_{\mathrm{U}}, \mathrm{SE}_{\mathrm{R}}\right)$ analysis of variance. The only main effect to reach significance was that attributable to target position, $F(1,36)=86.54$, $p<.001$. This effect is consistent with Foss and Lynch's results and indicates that the targets that occur late in SE sentences are responded to more slowly than those that occur early.

Although the main effect for Es did not approach significance, $F(1,36)=1.30$, $\mathrm{p}=.26$, the interaction between groups and Es was of marginal significance, $F(1,36)=3.50, p=.07$. While this suggests some caution in interpreting the present results, the fact that there was no other effect involving Es here or in any other analysis of this experiment suggests that this one marginal interaction was probably a chance effect.

The comparison of greatest interest is the interaction between target position and reduction, for the relative clause reduction effect should be reflected in this interaction. That is, there should be a difference between $S E_{U}$ and $S E_{R}$ when the target phoneme occurred late but not when it occurred early. This interaction yielded 
$F(1,36)=5.68, p=.02$. A Neuman-Keuls test (Winer, 1962) was performed among the four conditions involved in this interaction to further evaluate the locus of the effect. All pairwise comparisons were significant $(\mathrm{p}<.05)$, excepting that between reduced and unreduced $S E$ sentences with early target phonemes. Thus, late-occurring targets are responded to more slowly than early ones, consistent with the significant main effect for target position. More important, late occurring phonemes are responded to more slowly in reduced than in unreduced SE sentences.

The predicted three-way interaction between groups, target position, and reduction failed to materialize, $F(1,36)=0.03, p=.86$. It would appear, then, that the present results are inconsistent with those of Foss and Lynch and consistent with the other results on the relative clause reduction effect-reducing relative clauses results in increased comprehension difficulty. The fact that the three-way interaction was not obtained indicates that the failure of Foss and Lynch to obtain this effect cannot be attributed to the materials used in that study. We will retum below to the problem presented by those results.

Monitoring data for the RB sentences were analyzed separately. The main reason for this was that for the SE sentences an interaction was predicted that involved the groups variable which, for those conditions, however, the sentences were the same across groups and, hence, no effect involving this variable was expected. In addition, the reduction variable which was the focus of attention for the $\mathrm{SE}$ sentences was not meaningful for the RB sentences.

The mean speed scores for the RB sentences were subjected to a 2 by 2 by 2 , Groups by Target Position by Es analysis. None of the main effects or interactions approached significance. That target position did not yjeld a significant effect is consistent with the results of both experiments reported by Foss and Lynch and indicates that there is no increase in processing difficulty for $R B$ sentences late in the sentence.

In comparing the present data to those reported by Foss and Lynch, it should be borne in mind that Foss and Lynch analyzed their data somewhat differently. Specifically, all amalyses reported there were based on latencies for only those sentences comprehended correctly, whereas here all sentences were considered. Analyses comparable to theirs could not be performed on the present data because the comprehension criterion here was correct paraphasing rather than the fill-in-the-blanks task used by Foss and

Table 2

Mean Numbers of Propositions Recovered in the Paraphrasing Task

\begin{tabular}{lccccccc}
\hline & \multicolumn{3}{c}{ Group 1 } & & & \multicolumn{3}{c}{ Group 2 } \\
\cline { 2 - 5 } $\begin{array}{l}\text { Target } \\
\text { Position }\end{array}$ & RB & SE $_{\mathbf{U}}$ & SER & & RB & SEU & SE $_{\mathbf{R}}$ \\
\hline None & 14.65 & 16.15 & 15.90 & 16.90 & 19.25 & 18.10 \\
Early & 18.35 & 16.75 & 15.65 & 19.15 & 17.85 & 19.00 \\
Late & 15.85 & 13.70 & 13.00 & 18.45 & 16.00 & 14.25 \\
\hline
\end{tabular}

Lynch. The paraphrasing task is sufficiently difficult so that analyzing only those sentences correctly paraphrased would have caused us to discard more than $75 \%$ of the data. However, Foss and Lynch reported that their conclusions were not modified materially by using all of their data, so it would appear that this difference between experiments in data analysis cannot account for the difference in results.

\section{Paraphrasing Task}

The paraphrases were transcribed from the tapes made during the experiment. They were scored, independently and without knowledge of Ss' group membership, by two scorers using the criteria developed by Fodor and Garrett (cf. Fodor, Garrett, \& Bever, 1968). Paraphrase accuracy scores for the sentences in each condition were computed by summing the propositions accurately paraphrased across the 10 sentences in each condition for each $S$. The means of these scores are presented in Table 2.

The accuracy scores for the SE sentences were subjected to a 2 by 2 by 3 by 2 , Groups by Es by Target Position (E, L, N) by Reduction $\left(S E_{U}, S E_{R}\right)$ analysis. The only significant main effect was that attributable to target position, $F(2,72)=43.66, p<.001$. This indicates that paraphrasing was less accurate when the target occurred late in the sentence than when it occurred early or did not occur at all and suggests that the paraphrasing and phoneme monitoring tasks interact in their effects. That is, it appears that responding to a target phoneme late in an SE sentence may interfere with comprehension of the sentence, at least as reflected in paraphrasing. It is also possible that this effect is attributable to differences in the comprehensibility of the $\mathrm{SE}$ sentences assigned to the different target-position conditions.

The main effect for reduction did not achieve significance, $F(1,36)=2.96$, $p=.09$, indicating that relative clause reduction did not consistently reduce comprehension. That there is some effect for reduction is suggested by the fact that the Groups by Target Position by Reduction interaction also approached significance, $F(2,72)=2.82, p=.06$. That the reduction effect did not achieve significance is, of course, inconsistent with the results obtained by Fodor and Garrett and by Hakes and Cairns for the paraphrasing task. It is also inconsistent with the results for phoneme monitoring obtained here and by Hakes and Cairns. We will return to consider this inconsistency later.

The accuracy scores for the RB sentences were subjected to a separate 2 by 2 by 3 , Groups by Es by Target Position analysis. The only effect to reach significance was that attributable to target position, $F(2,72)=12.21, p<.001$. Unlike the target position effect for the $S E$ sentences, the effect here is mainly attributable to less accurate paraphrasing of those sentences not containing target phonemes. It seems likely that this effect is attributable to differences in the comprehensibility of the $R B$ sentences assigned to different target position conditions.

In summary, then, it would appear that the findings of the present experiment are generally consistent with those of Fodor and Garrett and of Hakes and Caims-reducing relative clauses tends to make SE sentences more difficult to comprehend. This effect is clearly demonstrated by the monitoring data and suggested by the paraphrasing data. Two problems remain: (1) the failure of the Foss and Lynch experiment to yield the reduction effect; and (2) the marginal effect in the present paraphrasing data.

We hypothesized that Foss and Lynch's failure to obtain the relative clause reduction effect was attributable to differences between the SE sentences that appeared in reduced and unreduced form in their experiment. This prediction was, of course, not borne out. The reduction effect appeared for the condition replicating Foss and Lynch's experiment (Group 1) as well as for the condition in which the sentence assignments were reversed (Group 2). At this point we have no explanation to offer for their results. It would appear that their finding of no difference attributable to reduction is just a Type II statistical error.

The fact that the paraphrasing data here yielded weaker evidence of the reduction effect than the monitoring data is consistent with the results of Hakes and Cairns's experiment, the only other 
experiment to directly compare the two tasks. The weaker effect supports their contention that paraphrasing is a less sensitive measure of comprehension difficulty than monitoring. Nonetheless, it is somewhat disturbing that the paraphrasing effect here was only marginal.

That the effect did not achieve a satisfactory level of significance may be partly attributable to the sentences used. It is apparent that for the sentences used here a far smaller proportion of the propositions were accurately paraphrased than were in the Hakes and Cairns experiment. There were some sentences here which no $S$ paraphrased correctly, and others where no $S$ accurately paraphrased anything but the matrix sentence. In addition, examination of the sentences and paraphrases suggests that several of the sentences were (close to) nonsensical. For example, (4) would appear to be, at best, a non sequitur, there being no apparent relation between the matron's being scared by the cat that the chauffeur had kicked and her believing the gypsy.

(4) The matron who the cat that the chauffeur kicked scared believed the gypsy.

It should be noted that Fodor and Garrett eliminated one sentence from their analyses on just the ground that their Ss found it nonsensical, and that sentence was not included in the Hakes and Cairns's experiment for the same reason. It seems reasonable that had the Ss here been asked, they would have rendered similar judgments on a number of sentences. Eliminating such sentences from the analyses might well have increased the magnitude of the relative clause reduction effect. That is, if a sentence is incomprehensible even when the relative pronouns are present, deleting them could hardly have a deleterious effect.

Another feature of the paraphrasing data is worthy of mention. There was a marked tendency for Ss to attempt to paraphrase the SE sentences by changing them into their RB form. For example, many Ss attempted to produce paraphrases of (5) with the form of (6).

(5) The puzzle (that) the youngster (who) the tutor taught devised bewildered the mathematicians.

(6) The tutor taught the youngster who devised the puzzle that bewildered the mathematicians.

Many attempts that appeared to involve this strategy were unsuccessful. And since such incomplete and incorrect paraphrases cannot be unequivocally interpreted as attempts to produce right-branching constructions, it is difficult to estimate just how prevalent this strategy was.

However, what is remarkable is not that such a strategy was prominent here but rather that it was prominent only here. There were only isolated cases of such paraphrases in Fodor and Garrett's data (Garrett, personal communication) and in Hakes and Cairns's data. Nor were they a notable feature of the paraphrasing data of an unpublished experiment on active and passive self-embedding by Forster, Garrett and Hakes. In addition, Blumenthal (1966) did not note any such strategy in his paraphrasing data for triply SE sentences. Blumenthal notes, rather, that Ss attempted to interpret all the relative clauses as referring to the initial subject noun phrase, a strategy which does not appear in any other paraphrasing data with which we are familiar.

The right-branching strategy that appeared here but nowhere else is undoubtedly attributable to the fact that in the present experiment all sentences that were not self-embedded were right-branching. The presence of $30 \mathrm{RB}$ sentences clearly provided a model which Ss attempted to adopt in dealing with the SE sentences. That such context effects have not appeared in other experiments reflects the fact that in those experiments SE sentences were either the only kind presented or were embedded among fillers of diverse structure. This context effect is reminiscent of that reported by Mehler and Carey (1967) and suggests that any inferences made from paraphrasing data about processes involved in comprehending and paraphrasing must take into account the composition of the entire set of sentences that are presented.

A further effect of this paraphrasing strategy may be noted in the paraphrases for the RB sentences. It is apparent from Table 2 that paraphrases for the RB sentences were not substantially more accurate than those for the SE sentences. This is a somewhat surprising finding, for it is generally agreed that $\mathrm{RB}$ sentences are considerably easier to comprehend than $\mathrm{SE}$ sentences. Examination of the errors made in paraphrasing the $\mathrm{RB}$ sentences indicates that a high proportion of the errors (at least under the Fodor-Garrett scoring criteria) involved $S$ repeating the sentence verbatim, a kind of error that was nearly nonexistent for the SE sentences. It would appear that the strategy of constructing right-branching paraphrases was so strong that it was applied to the RB sentences as well as to the SE sentences.

In conclusion, then, it would appear that the present results support the conclusions reached by Fodor and Garrett and by Hakes and Cairns. The relative-clause reduction effect appears to be a genuine one. Further, it would appear that comprehension-difficulty effects are reflected in both the phoneme monitoring and paraphrasing tasks, though more strongly in the former than in the latter. The failure of Foss and Lynch to obtain the reduction effect with the monitoring task should be discounted.

\section{REFERENCES \\ BLUMENTHAL, A. L. Observations with self-embedded sentences. Psychonomic Science, 1966, 6, 453-454. \\ FODOR, J. A., \& GARRETT, M. Some syntactic determinants of sentential complexity. Perception \& Psychophysics, 1967, 2,} 289-296.

FODOR, J. A., GARRETT, M., \& BEVER, T. G. Some syntactic determinants of sentential complexity, II: Verb structure. Perception \& Psychophysics, 1968, 3, 453-461.

FOSS, D. J. Decision processes during sentence comprehension: Effects of lexical item difficulty and position upon decision times. Journal of Verbal Learning \& Verbal Behavior, $1969,8,457-462$.

FOSS, D. J., \& LYNCH, R. H., JR. Decision processes during sentence comprehension: Effects of surface structure on decision times. Perception \& Psychophysics, 1969, 5, 145-148.

HAKES, D. T., \& CAIRNS, H. S. Sentence comprehension and relative pronouns. Perception \& Psychophysics, 1970, 8, 5-8.

MEHLER, J., \& CAREY, P. Role of surface and base structure in the perception of sentences. Journal of Verbal Learning \& Verbal Behavior, $1967,7,335-338$

WINER, B. J. Statistical principles in experimental design. New York: McGraw-Hill 1962.

\section{NOTES}

1. The present research was supported in part by NSF Grant GB-3629 to David T. Hakes and Robert K. Young and in part by NSF USDP Grant GU-1598 to Donald J. Foss. The authors wish to thank Barbara Tabor and Ben Dowell for their assistance in collecting data.

2. Address: Department of Psychology, The University of Texas at Austin, Austin, Texas 78712 .

3. As used in the present context, the term "surface structure" should not be construed in its technical linguistic sense. That is, neither Fodor and Garrett nor the present authors intend to imply that $S$ must construct a full labeled bracketing in order to find and utilize cues to the sentence's underlying grammatical relations. The representation in which such cues are discovered is probably more superficial and impoverished than the term "surface structure" implies.

(Accepted for publication April 3, 1970.) 